\title{
Minería como factor estructurante de la organización espacial de los barrios de origen ilegal en la localidad de Ciudad Bolívar
}

\author{
Structuring mining as a factor of spatial organization of illegal \\ origin areas in town Ciudad Bolivar
}

Linda Irene Gómez Fernández ${ }^{1}$

\section{Resumen}

La actividad minera ha sido históricamente uno de los elementos preponderantes en el desarrollo y organización espacial del área urbana de la localidad de Ciudad Bolívar (Bogotá, Colombia). Evidencia de esto, es la distribución espacial de algunos barrios de origen ilegal o informal, muy cerca de la minería o incluso en antiguas canteras, que configuran así, patrones comunes como el tipo de vivienda, la infraestructura vial e incluso dotacional.

El objeto de estudio es la configuración espacial de la localidad durante los años de 1970 al 2010 y su relación con la minería y los desarrollos de origen ilegal. El método investigativo utilizado, el análisis espacial, permite caracterizar los componentes del espacio y las relaciones entre estos, así como identificar algunos elementos que determinan el crecimiento urbano y sus tendencias.

Palabras clave: Barrios de origen informal, Ciudad Bolívar, Geografía Urbana, Minería. 


\section{Abstract}

Mining has historically been one of the major elements in the development and spatial organization of the urban area of the town of Ciudad Bolívar (Bogotá Colombia). Evidence of this is the spatial distribution of some neighborhoods of illegal origin or informal, close to mining or even in old quarries, setting well, common patterns and the type of housing, roads and even dotacional.

The analysis of the spatial configuration of the site during the years of 1970 to 2010 and its relation to mining and illegal source developments is the object of study. The research method used spatial analysis to characterize the components of the space and the relationships between them, enabling identify some elements that determine urban growth and trends.

Keywords: casual home neighborhoods, Ciudad Bolívar, mining, urban geography. 


\section{Introducción}

En este estudio para establecer el crecimiento urbano se consideraron variables cualitativas, entre las que se destacan por su sencillez y disponibilidad de información, elementos físicos susceptibles de ser medidos y cuantificados como el tamaño, la forma, la distribución en la unidad de análisis de la actividad minera y la relación entre los mismos. Para el análisis de la información se consideró como la unidad mínima los desarrollos progresivos o barrios de origen informal ${ }^{2}$.

Por lo general, los barrios de origen ilegal se originan respondiendo a la necesidad de localización de una población determinada, asociada a alguna actividad económica o responden a algún requerimiento funcional, espacial o relación con su entorno, determinando la forma de crecimiento y el emplazamiento de la población.

La hipótesis de estudio plantea que en la localidad de Ciudad Bolívar el surgimiento, desarrollo y consolidación de los barrios de origen informal se vinculan con la extracción minera remplazando o llenando espacios dejados por la actividad, y generan-

2 Los barrios se clasifican de acuerdo al origen en Legales e llegales: Los barrios Legales surgen como consecuencia de alguna política, programa o intervención del Estado; y los de origen ilegal surgen espontáneamente por gestión de las comunidades sin responder a ninguna estrategia de desarrollo planificado. do efectos en los ámbitos ambiental, ecológico, social y económico ${ }^{3}$. Por tanto, la ciudad requiere conocer las relaciones entre la minería y el crecimiento urbano, y las tendencias de ocupación alrededor de la actividad minera, para poder planificar la intervención en el territorio y tomar medidas de control adecuadas.

\section{2. Área de estudio}

Bogotá D.C., capital de la República, se localiza en la vertiente occidental de la cordillera Oriental de los Andes colombianos, en el suroriente del departamento de Cundinamarca ${ }^{4}$. Según lo establece el Acuerdo 2 de 1992, se subdivide en 20 localidades. La zona urbana se ubica principalmente en la Sabana de Bogotá a una altura promedio de $2600 \mathrm{msnm}$. (SDA, 2007, p. 26- 27).

Ciudad Bolívar es la localidad número 19 y se encuentra en el sur de la ciudad de Bogotá, en la cuenca del río Tunjuelo. Posee una extensión territorial de 13.000,3 ha, de las cuales el 24,91\%, es decir 3.239,8 ha, corresponde a suelo urbano. (SDP, 20011, p.10). (Figura 1)

3 Según Camargo (2005) este proceso de sucesión de sistemas de alteridad configura una dinámica que se repite en la ciudad no necesariamente en torno a la minería pero si alguna actividad económica.

4 Entre los $03^{\circ} 44^{\prime}$ y los $04^{\circ} 49^{\prime}$ de latitud norte y los $74^{\circ} 00^{\prime}$ y los $74^{\circ} 28^{\prime}$ de longitud oeste. 


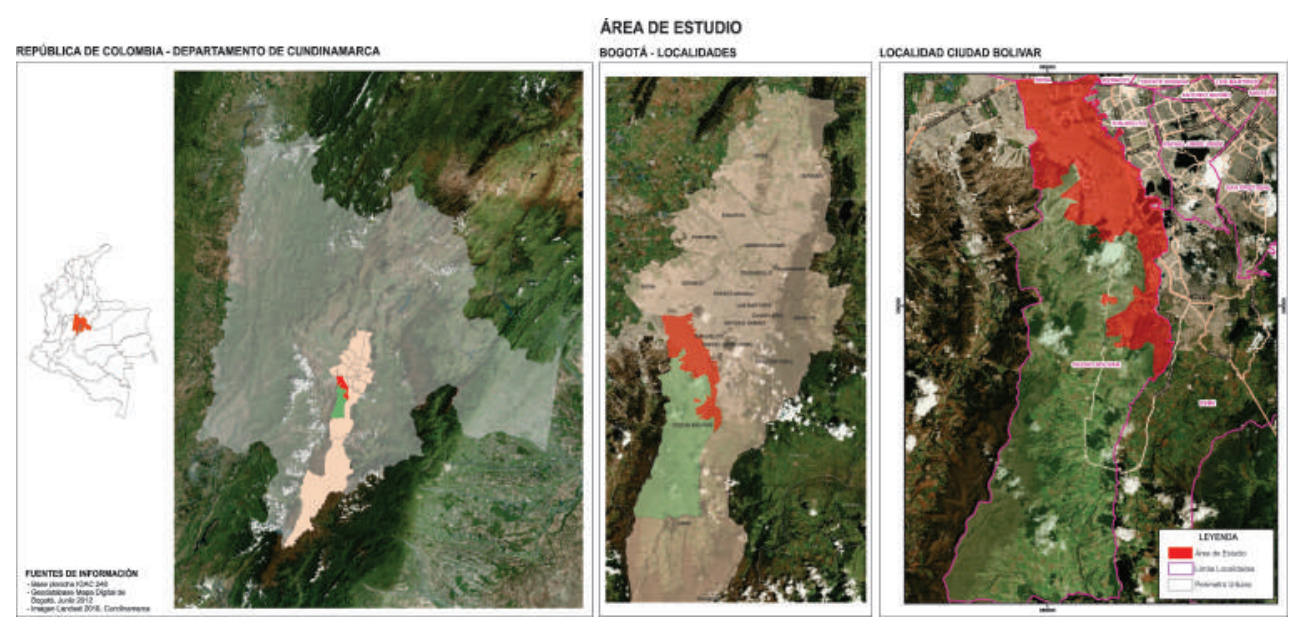

Figura 1. Localización general del área de estudio

Fuente: Imagen de Satélite Google Earth, Vectores Mapa base Unidad Administrativa Especial de Catastro Distrital, 2013

La localidad presenta pisos térmicos de frio semi árido (sic) a frío semi húmedo (sic), con temperaturas entre los 19 a $9^{\circ} \mathrm{C}$ con promedio de $14^{\circ} \mathrm{C}$; la precipitación oscila entre los 500-800 $\mathrm{mm}$ al año. Las formaciones geológicas que afloran en la localidad están constituidas por rocas sedimentarias (tanto de origen marino como continental) y depósitos no consolidados que presentan edades que van desde el Cretácico Superior hasta el presente. Se identifican cuatro ambientes morfológicos principales: Ambiente Fluvial, Estructural, Denudativo y Antrópico; suelos característicos de montaña estructural erosional de clima frío húmedo, estos se destacan por la presencia de los Alfisoles de tendencia ústica, y por sus horizontes argílicos bien desarrollados sobre depósitos aluviales y coluviales. Por el alto nivel de intervención en la zona, la cobertura natural como la vegetación riparia, matorrales y pajonales subxe- rofíticos son escasos y se concentran en la parte alta de la zona urbana y en la ronda hídrica de algunas de sus quebradas. Hace parte de la cuenca baja del río Tunjuelo. En la localidad se identifican cuatro subcuencas: quebrada Yerbabuena, Trompeta, Zanjón de la Estrella y Limas, las dos últimas se encuentran intervenida en más del $80 \%$. Las quebradas Limas y Trompeta aún se conservan en su parte alta. (SDA, 2007, p. 38-51)

\section{Marco teórico-metodológico}

En este documento se consideran aspectos fundamentales de la geografía urbana, como el discurso de la ecología urbana ${ }^{5}$, cuyos planteamientos se

\footnotetext{
5 Se percibía como contraria al positivismo, ya que no concebía el estudio de la geografía a partir de métodos y prácticas inherentes a la ciencia natural a partir de leyes y teorías. Sin embargo, una de sus principales críticas era la naturalización de la sociedad (Berdoulay, 1998, citado por Montoya, 2009).
} 
originan en la Escuela de Chicago. Este discurso tomó fuerza a comienzos del siglo XX y su objetivo era el estudio del comportamiento y adaptación del hombre a la naturaleza. Park (citado por Montoya, 2009, p. 151) plantea la ciudad como el hábitat natural del hombre civilizado, e identifica problemáticas asociadas a aspectos culturales como la pobreza, la marginalidad, la inmigración de la población, la relación hombreambiente urbano, los cuales hoy en día persisten y son de gran interés en los estudios urbanos.

Diversas corrientes epistemológicas confirman el papel preponderante de la ciudad en la geografía urbana. Anthony Sutcliffe, (1981, p. 373, citado por Almandoz, 2003), señaló que la historia urbana es, en buena medida, la historia de la construcción de la ciudad, cuyo elemento más específico viene dado por su énfasis en la forma física.

Esto coincide, desde el punto de vista teórico, con las definiciones que se han dado de lo urbano, las cuales son básicamente de dos tipos. Por un lado se encuentran las que se basan en el tamaño y las funciones económicas que se desarrollan y en menor proporción la forma y el aspecto de la conglomeración. Por otro, se hallan las definiciones eclécticas, que intentan dar una idea de la complejidad de lo urbano sintetizando las diversas características previamente definidas (Capel, 1975, p. 264).

Por eso, entender la ciudad desde la perspectiva funcional, obliga a considerar las actividades económicas que dentro de ella se desarrollan, no solo la industria y el comercio sino otros servicios especializados como transportes, educación, administración del Estado y la banca (Aurousseau, 1961, citado por Capel, 1975, p. 265). Esta mezcla de actividades sumada a la concentración de la población en un lugar determinado, dan cuenta de otra característica fundamental de las ciudades: la complejidad.

Por tanto, las ciudades se establecen, se desarrollan y desaparecen gracias a los flujos de materia y energía internos y externos, a través de los intercambios entre los diferentes sectores económicos y sociales (Díaz, 2011, p. 5), tales como las fuerzas de la industria, el comercio concentrado, la demanda de mano de obra, las relaciones de poder, las relaciones de intercambio y redistribución. También la ciudad, como si fuera un organismo más, necesita consumir energía y materia para cubrir las necesidades de sus habitantes: domésticas, de transporte, de alumbrado, de climatización, etc. (Camargo, 2005, p. 16).

Otra de las manifestaciones de la complejidad de la ciudad como sistema es la organización espacial urbana. La 
organización espacial sugiere una connotación geográfica, por cuanto es el resultado de la actividad del hombre en el espacio, (...) incluye el accionar histórico de la sociedad en el espacio, el proceso desigual y combinado de apropiación y mo- delamiento del territorio que sirve de soporte a la sociedad y el papel condicionante que cumple el espacio socialmente organizado con respecto a los procesos sociales (Massiris, 2005). (Figura 2)

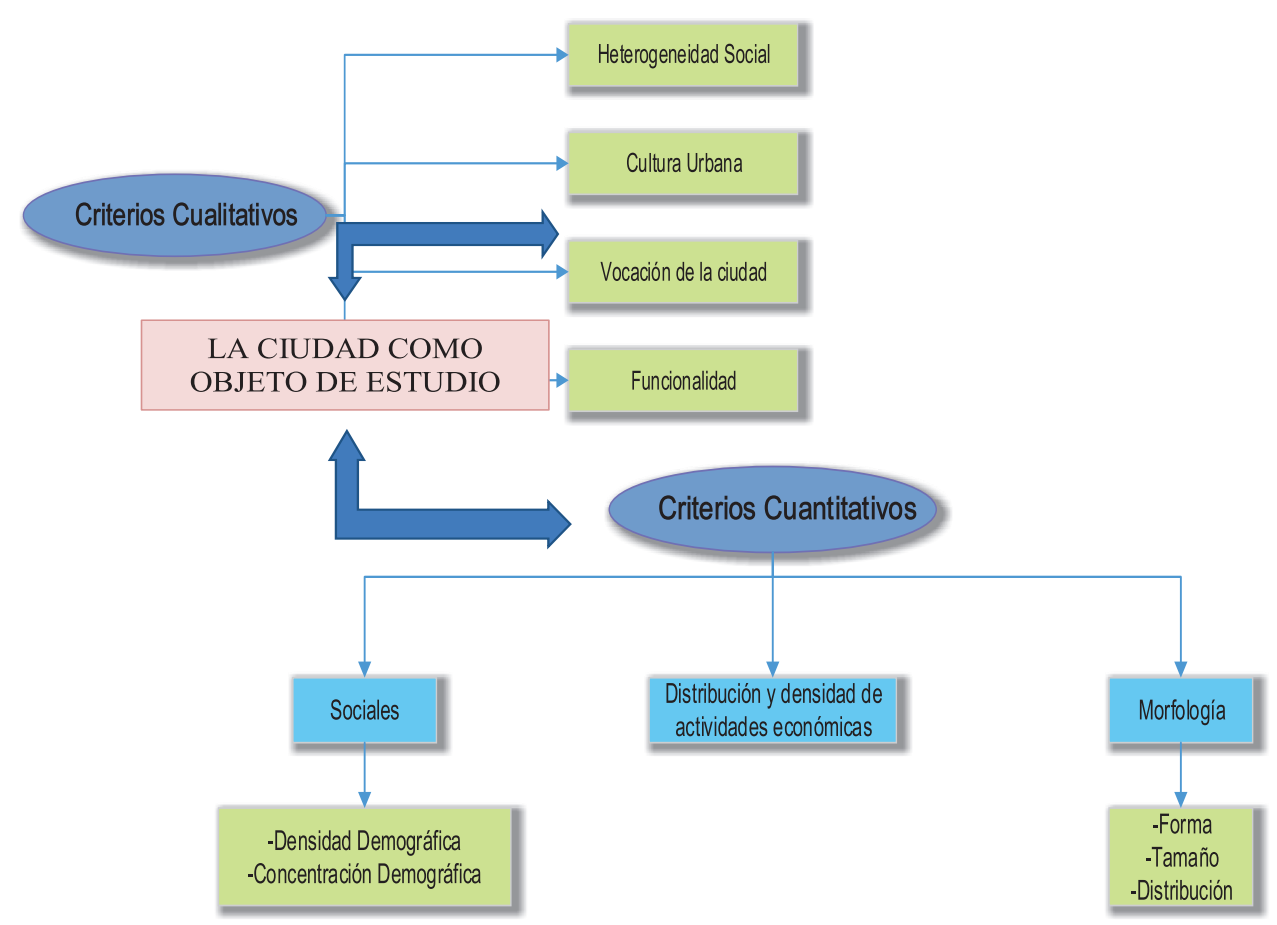

Figura 2 - La ciudad como objeto de estudio criterios cuantitativos y cualitativos Fuente: Adaptado de Massiris, 2005

La organización espacial se materializa en una estructura espacial que puede definirse como un arreglo de sistemas físicos que se vinculan con las actividades y prácticas humanas, delimitado funcionalmente por la utilización del espacio geográfico, el cual se transforma y reproduce en el tiempo. La estructura urbana es un producto físico y socioeconómico de una o varias estructuras espaciales.

Es así como las ciudades presentan coincidencias en la distribución de las distintas zonas y áreas del suelo conforme a una serie de factores, de los cuales se pueden destacar: la accesibilidad, el uso del suelo y el 
modo de efectuarse la expansión urbana, las características poblacionales, funciones y actividades económicas que vuelven atrayente y destacado un espacio geográfico.

En conclusión, podemos afirmar que las ciudades son espacios abiertos, complejos, relativamente autónomos, dinámicos y en expansión, en donde se localizan unos determinados elementos que se encuentran conectados.

La evolución de la Geografía Económica aporta una propuesta de análisis de espacios y lugares con intereses particulares, físicos y humanos, para poder cubrir las necesidades humanas empleando una serie de aspectos ligados a la producción, distribución y consumo de los bienes económicos. La geografía económica no puede ser separada de la geografía física, ya que la naturaleza, que es su objeto, suministra al hombre los elementos de la producción, ni tampoco de la geografía humana, porque las sociedades, que son su objetivo, organizan esta producción, la utilizan por medio del consumo y establecen los intercambios según las predisposiciones naturales" .... "La geografía económica trata, así pues, de las actividades productivas e intenta explicar por qué ciertas regiones destacan en la producción y exportación de determinados bienes y servicios y porqué otras sobresalen en su utilización o consumo". (Franquet, 2007, p. 40).

Como método investigativo se toman algunos elementos de la metodología de análisis espacial y otros de la metodología de los sistemas de alteridad. La posición teórica general del análisis espacial consiste en proponer una explicación parcial y posibilidades de previsión con respecto al estado y la evolución probable de los objetos-unidades geográficos, a partir del conocimiento de su situación en relación con los otros objetos geográficos (Ortiz, 2004, p. 2) (Figura 3).

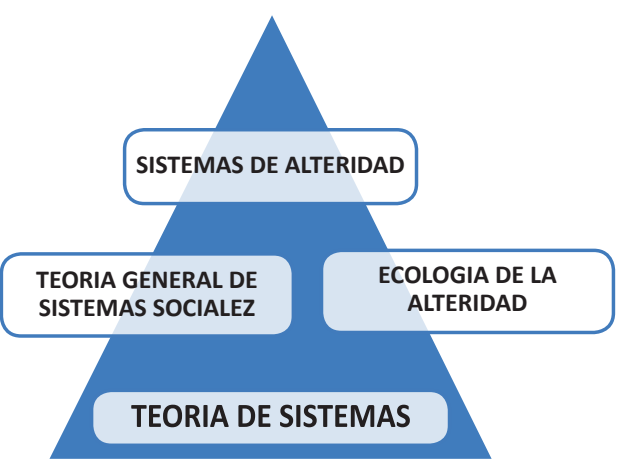

Figura 3. Método investigativo

Fuente: Adaptado de Ortiz, 2004 - Camargo, 2005 
Los métodos utilizados para el análisis espacial son muy diversos. El empleo de la estadística clásica conduce a olvidar en un primer momento la localización de los objetos y, completada por la cartografía, encuentra luego, en un análisis en términos de residuos, los efectos espaciales. Por el contrario, los métodos de la geoestadística y de la morfología matemática, agrupados en la estadística espacial, analizan directamente las informaciones geocodificadas. (Ortiz 2004, p. 1)
El sistema de alteridad es la unidad estructural-funcional del paisaje humanizado; es un modelo de la forma típica de relación (adaptación/ adecuación) entre un grupo humano socioeconómicamente diferenciado y su entorno, formulado según las propiedades y componentes de los sistemas autopoyéticos (abiertos autoorganizativos y autorreplicantes). (Camargo, 2004 citado en Camargo \& Guerrero, 2005, p. 98) (Figura 4)

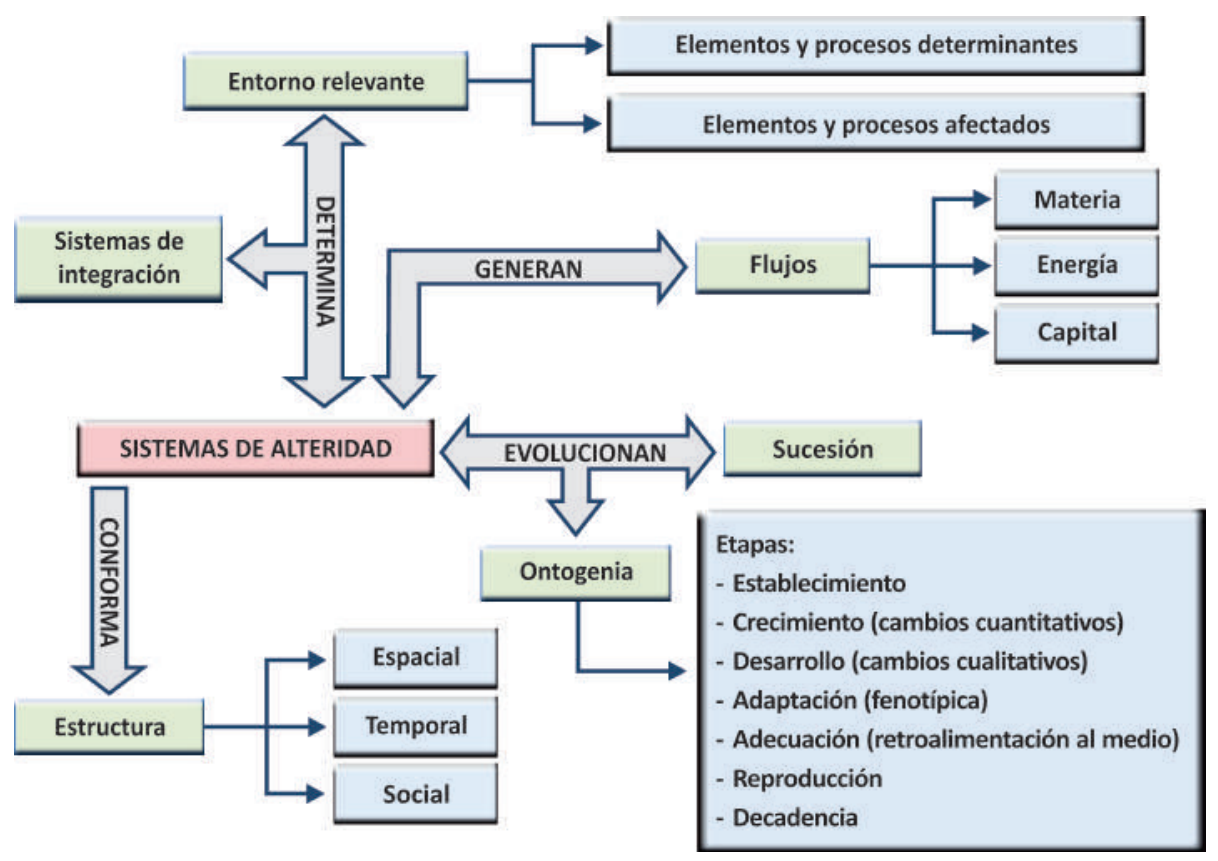

Figura 4. Estructura de los sistemas de alteridad

Fuente: Adaptado de Camargo, 2005

Como sistema abierto tiene su propio entorno, por ser autorreferenciado genera sus propias estructuras y se autorreproduce, y es autopoyético porque todo lo que sucede en el sistema corresponde a una operación, incluidas sus estructuras (Castellanos, 2003, p.5). 
La diferenciación del sistema está dada por "la repetición de la diferencia entre sistema y entorno de los sistemas. El sistema total se utiliza a sí mismo como entorno de la formación de sus sistemas parciales". (Luhmann, 1998, p. 31)

En el análisis de los sistemas de alteridad se entiende la alteración como

"El proceso ecológico desencadenado por una o más perturbaciones, a través de la cual se modifican la estructura, composición y/o la función de un ecosistema a través del encadenamiento y retroalimentación de distintos cambios determinados por las propiedades elementales (condiciones de la perturbación o del ecosistema pre-disturbio) y las propiedades emergentes (condiciones resultantes de la alteración misma y distintivas de los estados y dinámicas alterados).

Por tanto, la alteración no es simplemente el efecto o la suma de las perturbaciones, sino un típico proceso de reorganización autopoyética del ecosistema, en el cual se combinan procesos y efectos de perturbación, regeneración y respuestas más o menos individuales de distintos componentes del ecosistema." (Camargo \& Guerrero, 2005, p. 79).

Estudiar los procesos de ocupación y transformación del territorio des- de la perspectiva de los sistemas de alteridad como método, obliga a una aproximación entre las ciencias naturales y las ciencias sociales desde una visión compleja no lineal, donde el origen y evolución del sistema se asemeja al "bucle tetralógico" descrito por Morin (1999, p.71 citado por Castellanos, 2003), cuando señala que toda organización proviene del desorden y es producto de interacciones que la generan, para propender al orden sistémico, el que a partir de dispersiones posteriores y nuevas interacciones genera un nuevo orden distinto al anterior establecido. (Castellanos, 2003)

"La orientación espacial de la expansión (crecimiento y reproducción) de un sistema de alteridad depende principalmente de la oferta ambiental según los factores relevantes para el tipo de sistema en cuestión.... Los factores que condicionan la aparición inicial de un sistema de alteridad no siempre son los mismos que determinan sus posibilidades de crecimiento y reproducción. Por eso a veces es necesario diferenciar entre factores de establecimiento y factores de expansión". (Camargo \& Guerrero, 2005, p. 107)

En un mapa de anisótropas estas "condiciones pueden identificarse unas direcciones en las cuales las líneas isótropas se dilatan (puntos más distantes se hacen más atracti- 
vos) y otras en las cuales las isótropas se comprimen (puntos más cercanos pero poco atractivos). Las primeras las identificamos como vectores de ocupación, mientras las segundas representan barreras a la ocupación". (Camargo \& Guerrero, 2005, p. 109)

\section{Desarrollo investigativo}

En el área del Distrito Capital y específicamente en la zona sur donde actualmente se ubica la localidad de Ciudad Bolívar, la actividad minera se consolida a mediados del siglo XX dada la configuración litológica del área que permite la exploración y producción de gravas y arenas ${ }^{6}$ para el concreto, que surgieron en la década de los treinta con la denominada "Compañía de Concretos y Triturados Ltda.”. En 1945 fue adquirida, incluyendo todos los terrenos y maquinarias, por Fábrica de Cemento Samper (Secretaría Distrital de Ambiente -SDA- \& Universidad Nacional de Colombia, 2008, p. 90).

En el periodo comprendido entre 1950 y 1959, se inicia "La explotación intensiva de la actividad extractiva comenzó con la extracción de arenas del río Tunjuelito y la quebrada Limas, luego se comienza a intervenir las áreas de peña y finalmente, en un tercer momento, se ini-

6 Este tipo de materiales está asociado a riveras, cauces y ríos. cia la extracción de arcillas" (Rayo, 2008, p. 22).

El análisis de las anisótropas generadas por la minería nos muestra como la minería en el periodo 1940 a 1970 tiende al crecimiento sobre la UPZ Ismael Perdomo y la quebrada Trompeta y Limas. Para el final del periodo se identifican (Lozano, 2008): (Figura 5)

- 88 polígonos de explotación, existentes en la década del cincuenta; 37 permanecen para la década del 60 , es decir el $42 \%$. Por tanto, de los 164 existentes en la década del 60, 127 son nuevos polígonos de explotación.

- El área ocupada por la actividad minera aumenta de 66,30 ha. en la década del 50 a 89,85 ha. en la década del 60.

- Permanecen 16 polígonos de la década del 40 (tres canteras y trece chircales)

- Los siete desarrollos barriales que surgen en la década del 50: México, Tabor Altaloma, Ismael Perdomo Las Lomas, Buenos Aires, San Francisco, Potreritos e Inés Elvira, son legalizados en el año de 1963. En la misma zona (cuenca Zanjón de la Estrella) se inicia el desarrollo de los barrios San Fernando, República de Venezuela, El Consuelo y El Salitre. (SDA, 2007) 

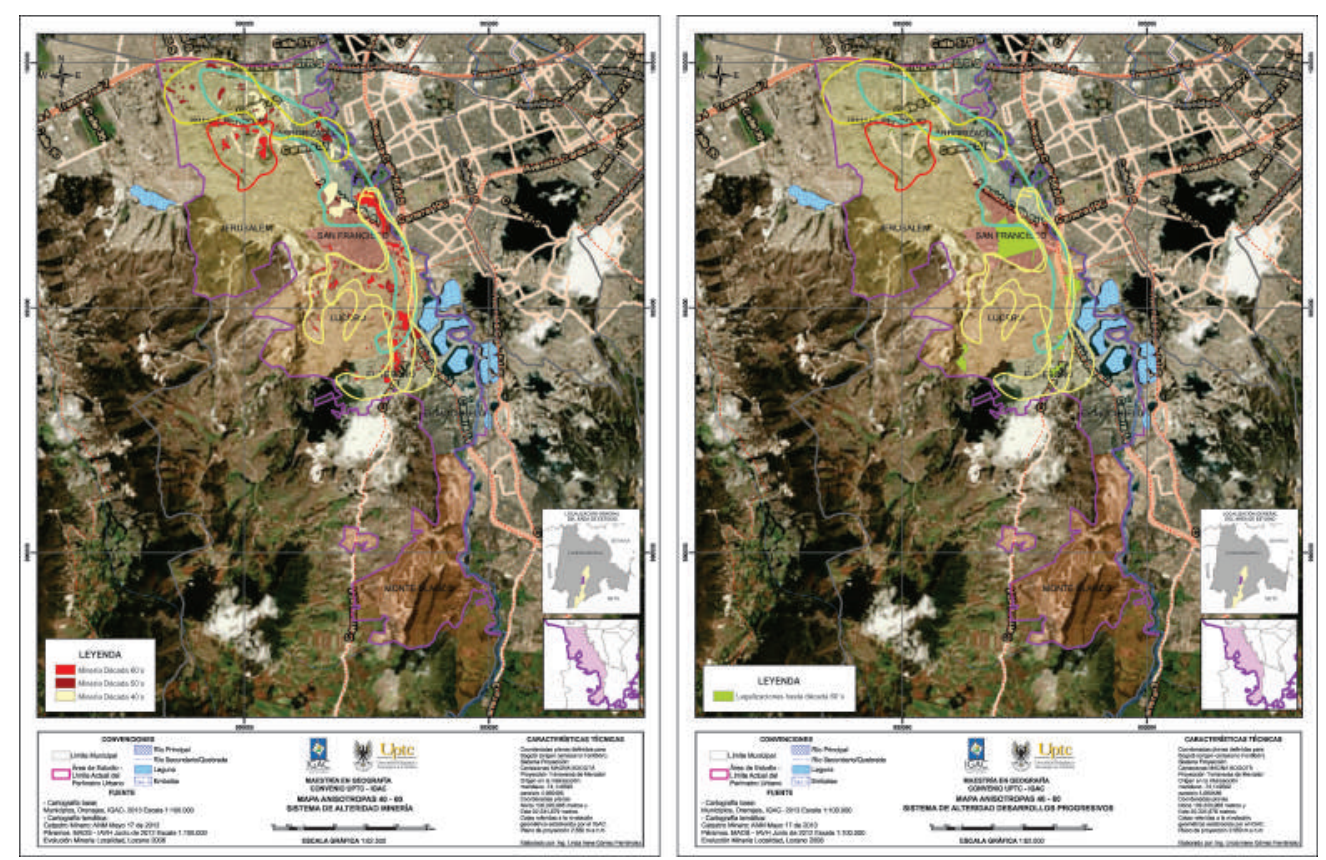

Figura 5. Ambiente anisotrópico antes del año 1970. Crecimiento urbano.

Fuente: Anisótropas Gomez, 2013 - Inventario Minero Lozano, 2008 actualizado ANM, 2013 (títulos mineros) Cartografía Base UAECD, 2013

A finales de los años setenta y principios de los años ochenta se consolidó la planificación y construcción de la avenida Boyacá-autopista Villavicencio, lo que potenció la valorización y edificación de los terrenos aledaños a este eje vial.

Según Lozano (2008) la distribución espacial de la actividad minera para los años setenta presenta dos núcleos importantes: uno alrededor del barrio Ismael Perdomo - La Loma (legalizado en 1963) en zona de montaña, donde se ubican 15 polígonos de desarrollo minero avanzado a moderado y en menor proporción actividad minera artesanal. El otro núcleo es en la cuenca de la quebrada Limas donde se ubican 26 canteras de menor tamaño pero que muestran la presión sobre el recurso. Los chircales suman un total de 43, ocupando un área de 51,05 ha. El polígono de mayor área se localiza al norte del barrio San Francisco sobre la rivera de río Tunjuelo que se incrementa de un área de explotación de 18,61 ha. En los 60's a 26,58 ha., para el periodo de 1970 a 1979. En estos chircales se explotan arcillas, y se consideran en su mayoría de desarrollo incipiente, 9 se encuentran en desarrollo avanzado, destacándose los chircales próximos al actual barrio El Consuelo en la cuenca de la 
quebrada Zanjón de la Estrella que presenta desarrollo muy avanzado. La altura de explotación está en el rango de 2 a 9 metros, los impactos se consideran bajos a muy bajos.

El desarrollo de los barrios va evolucionando alrededor de los núcleos de explotación y se legalizan cuatro barrios: El Salitre (1971), El Consuelo (1975), República de Venezuela (1979) y San Fernando (1974). Se encuentran en desarrollo los barrios La Cabaña, Perdomo Alto, México III Sector, Cooperativa Ismael Perdomo, Alfa, San Isidro, La Esperanza, Buenavista Sector A, Sotavento, Santa Inés La Acacia, Bella Vista Lucero Alto, San Joaquín, San Luis Altos de Jalisco, Parcelación Bogotá y El Castillo (SDP, 2007)

Con relación a la actividad minera, para 1985 y de acuerdo con el censo de la industria extractiva realizado por Estudios y Proyectos Ambientales y Mecánicos S.A. - EPAM S.A., se reportan 684 minas en Bogotá (Secretaría Distrital de Ambiente -SDA- \& Universidad Nacional de Colombia, 2007, p.105).

Para la década del ochenta se legalizaron 16 barrios más, para un total de 27 barrios formalizados, de los cuales cuatro quedan fuera del perímetro urbano. Para finales de la década, existían 117 desarrollos en trámite de legalización (Figuras 6a y 6b).
En el periodo 1990-1999, se presenta el avance más notorio en la legalización de barrios de la localidad, aunque estos no cumplían con todos los requerimientos de seguridad y calidad de vida. Sin embargo, con la consolidación de los antiguos barrios, se consigue un acceso formal a servicios como luz y agua, lo que indica una oportunidad para los pobladores de los nuevos barrios de tener una vivienda digna en el futuro. En este momento se ocupan todos los vacíos dejados entre los primeros barrios y la ciudad consolidada, de manera que la mancha urbana de Bogotá se extiende finalmente sobre la cuenca del Tunjuelo.

El DAMA (hoy Secretaría Distrital de Ambiente) realiza un censo minero en 1995, que arroja como resultado un total de 400 minas dentro del perímetro urbano de Bogotá. Para el área urbana de la localidad de Ciudad Bolívar se tienen 74 puntos con actividad minería. Ocupan 86,27 ha, de los cuales 60 son canteras y los restantes chircales; las canteras por su parte ocupan 55,16 ha y van desde 0,1 ha hasta 7,1 ha. En estado de abandono se encuentran 29 canteras, en desarrollo avanzado 4 y las restantes en desarrollo incipiente. Las 31 canteras que se encuentran en operación explotan arenas y causan impactos de moderados a bajos. Los chircales se concentran en el valle aluvial del río Tunjuelo y en la cuen- 


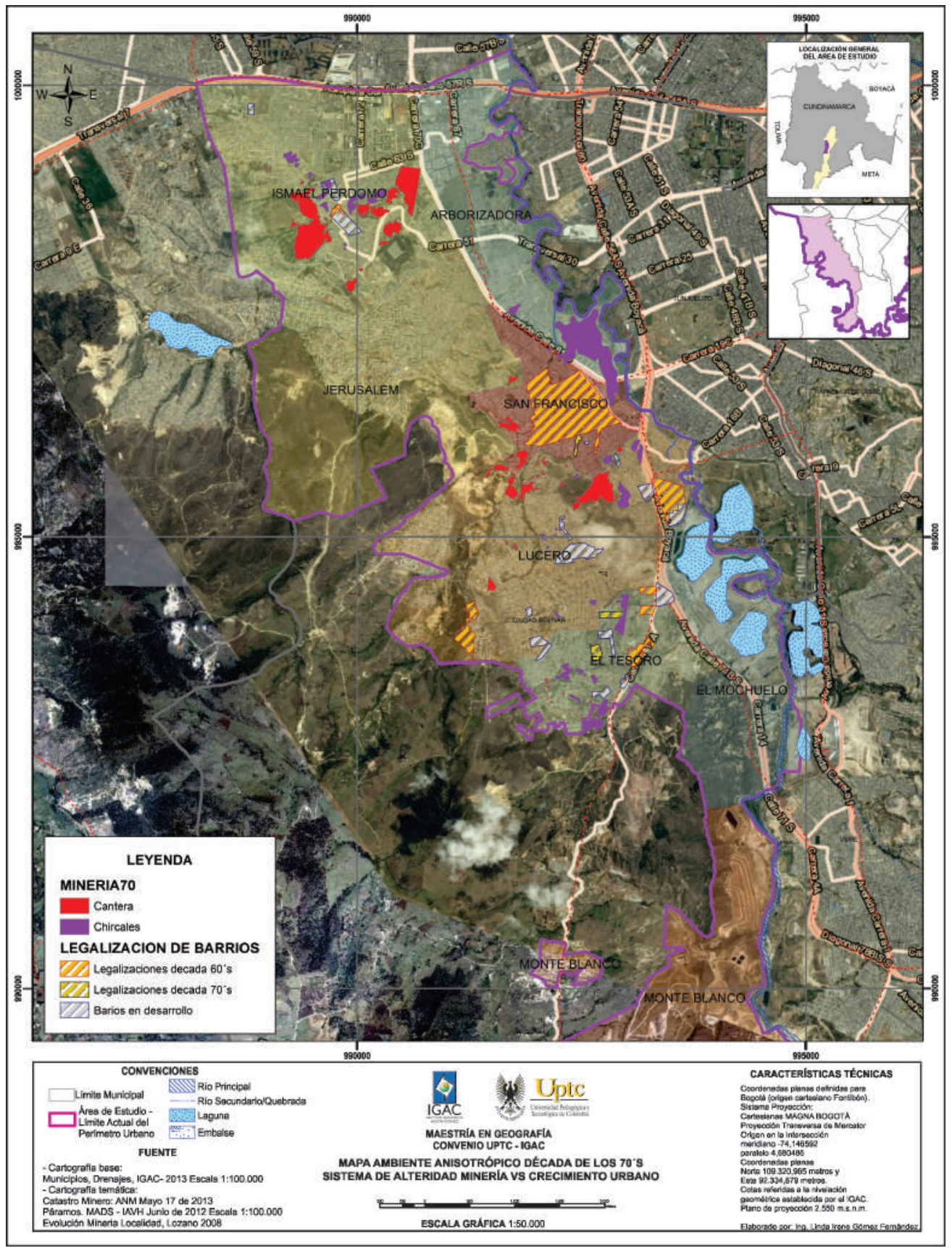

Figura 6a. Ambiente anisotrópico periodo 1970-1979. Minería vs crecimiento urbano.

Fuente: Anisótropas Gomez, 2013 - Inventario Minero Lozano, 2008 actualizado ANM, 2013 (títulos mineros) Cartografía Base UAECD, 2013 


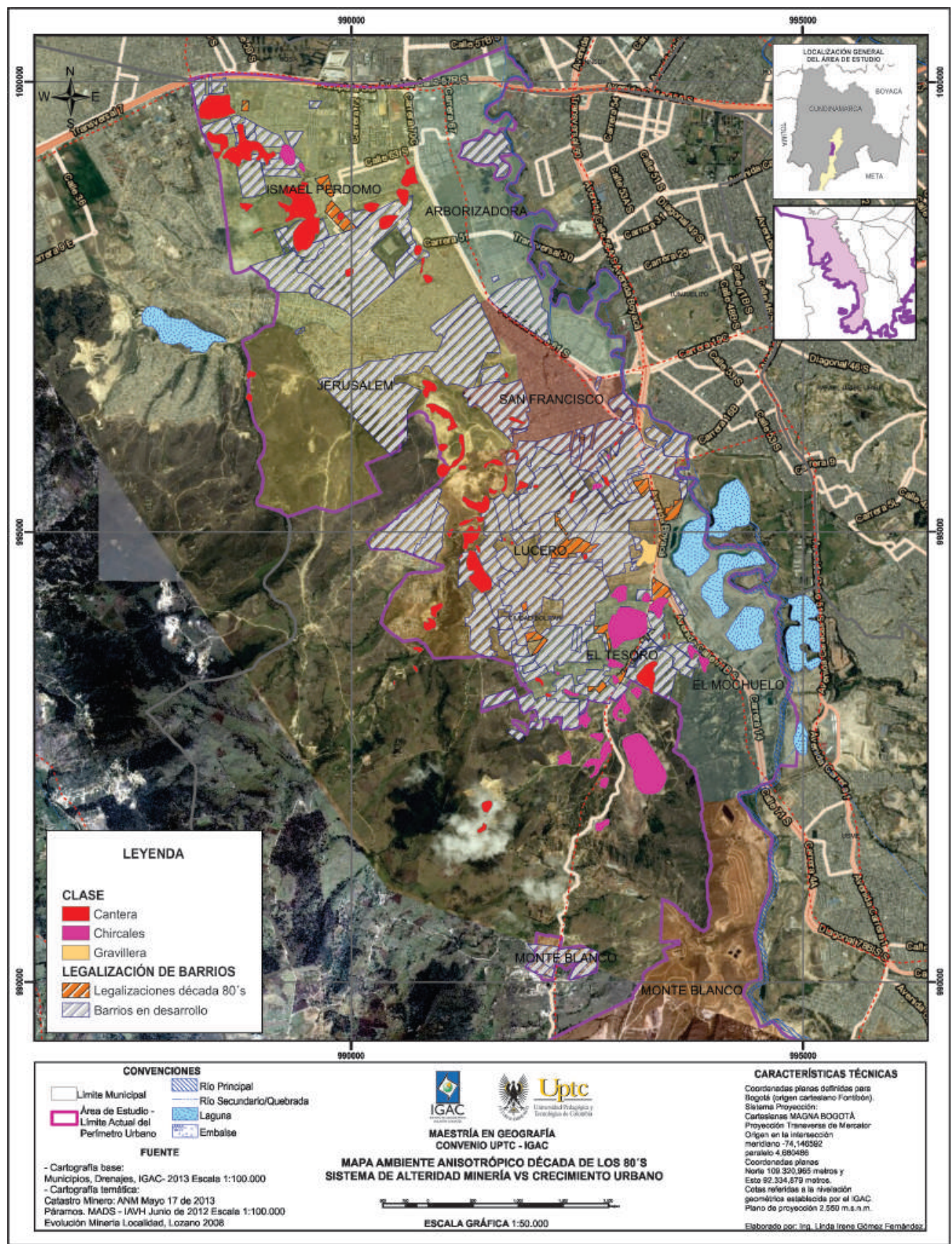

Figura 6b. Ambiente anisotrópico periodo 1980-1989. Minería vs crecimiento urbano.

Fuente: Anisótropas Gomez, 2013 - Inventario Minero Lozano, 2008 actualizado ANM, 2013 (títulos mineros) Cartografía Base UAECD, 2013 
ca de la quebrada Trompeta, cubriendo un área de 21 ha, los tamaños van de menos de 1 hasta 7 hectáreas, de impacto moderado a alto. En síntesis, para esta época la minería se encuentra totalmente inserta en la matriz urbana. (Lozano, 2008) (Figura 7a y Figura 7b)

A partir del año 2000, la dinámica de crecimiento de la ciudad se ve marcada por el Plan de Ordenamiento Territorial, periodo caracterizado por una política de planeación distrital mucho más estructurada.

Con la definición y establecimiento de los parques minero-industriales (PMI), a partir del Plan de Ordenamiento Territorial en el año 2000, se reglamentó su funcionamiento y administración por parte de las autoridades de planeación y ambiental del Distrito Capital. Sin embargo, la ciudad no cuenta con este instrumento de ordenamiento, y tampoco, con la decisión por parte de los mineros de unificar áreas a partir de su titularidad minera y concretar unidades mineras desde el punto de vista minero, ambiental, urbanístico, económico social y administrativo (Fierro, 2007, p. 9).

$\mathrm{Al}$ revisar la situación actual de la distribución espacial de la actividad minera se observa que del total de predios con actividad extractiva dentro del perímetro urbano de la localidad de Ciudad Bolívar, existen veintitrés
(23) predios ubicados en zona no compatible con la minería y que deben ser objeto de recuperación morfológica y ambiental. Para ello la Secretaría Distrital de Ambiente, durante los años 2007 a 2009 solicito los estudios de Plan de Manejo, Recuperación o Restauración Ambiental (PMRRA) para su evaluación y aprobación, además de realizar un seguimiento a la implementación de los mismos. De igual manera, ha requerido la suspensión de las actividades a aquellos predios que no presentaron el estudio (SDA, 2010)

Según la información reportada para el año 2009 por la Secretaría Distrital de Planeación, en la localidad hay inventariados 179 barrios de origen ilegal, que cubren 1.314 ha, lo cual representa el $41,04 \%$ del total del área de estudio. Las viviendas que surgen de la autogestión de sus habitantes, presentan déficit en varios aspectos físicos.

La estructura espacial de los barrios de origen ilegal se encuentra extremadamente ligada a la fase de "desarrollo" del sistema y para determinar las características morfológicas de los barrios se debe tener claro primeramente las etapas que constituyen dicha fase. Las cuales son tres etapas constitutivas de la fase de desarrollo del sistema: la etapa de establecimiento, la etapa de crecimiento y por último la etapa de consolidación, cada una 


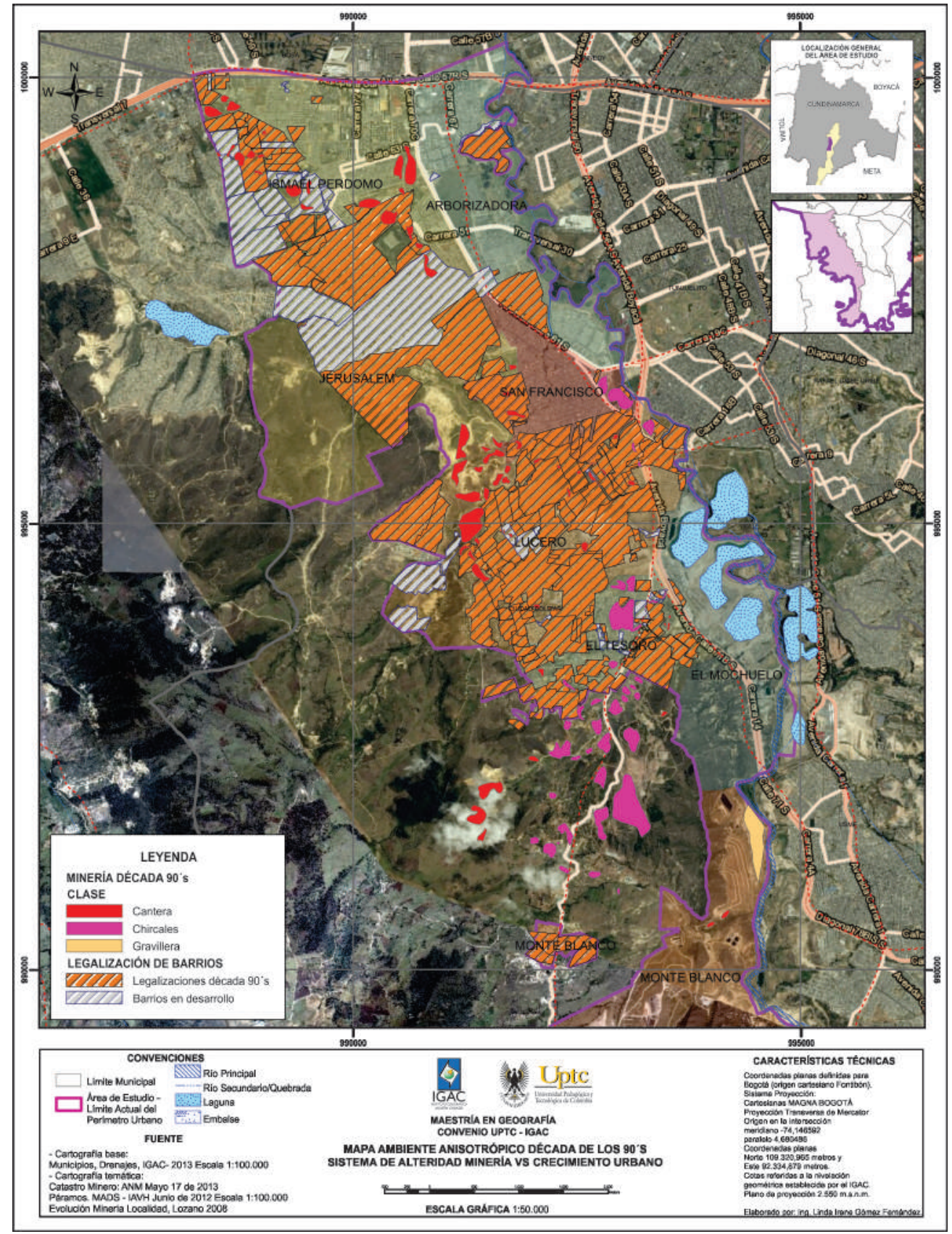

Figura 7a. Ambiente anisotrópico periodo 1990-1999. Minería vs crecimiento urbano

Fuente: Anisótropas Gomez, 2013 - Inventario Minero Lozano, 2008 actualizado ANM, 2013 (títulos mineros) Cartografía Base UAECD, 2013 


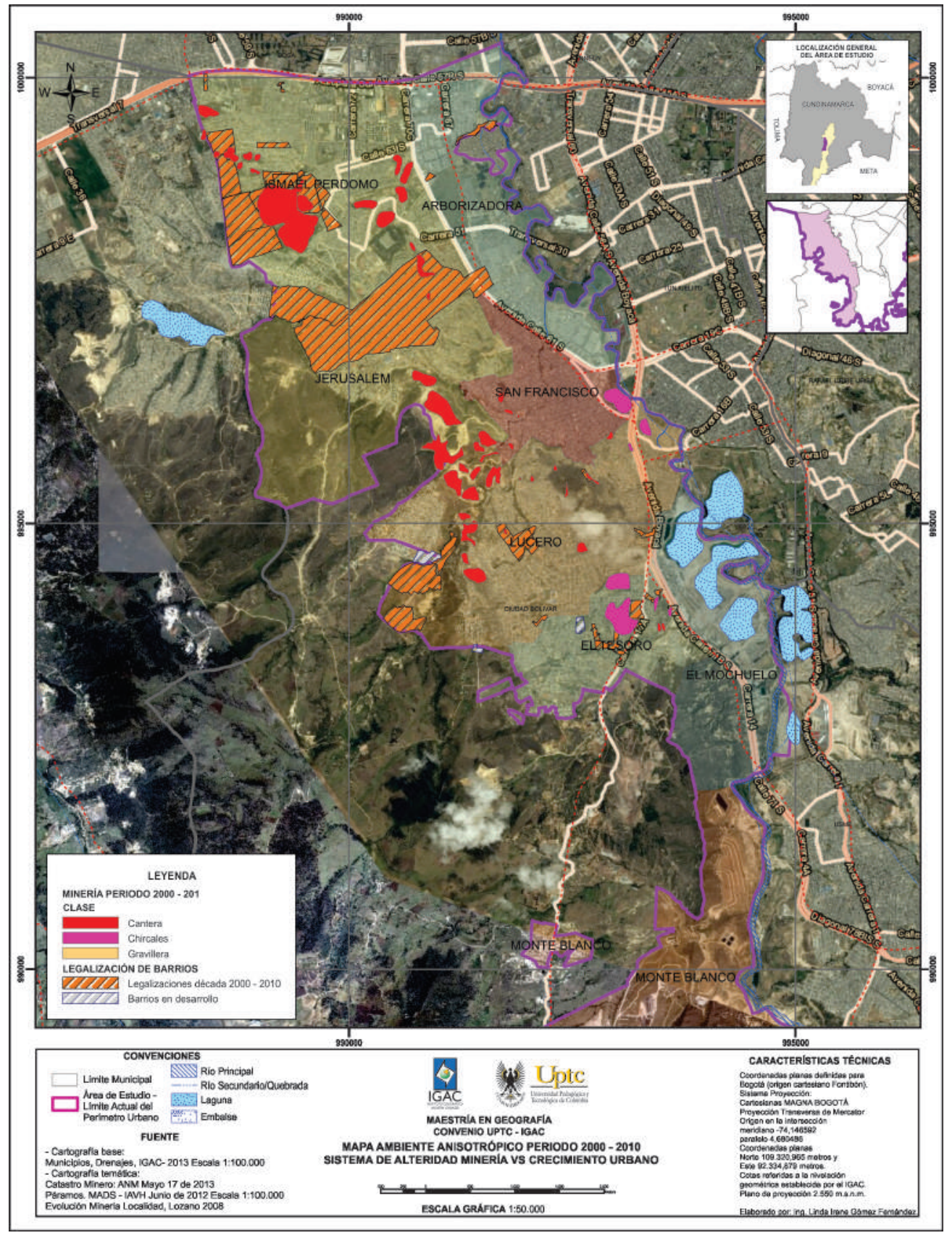

Figura 7b. Ambiente anisotrópico periodo 2000-2010. Minería vs crecimiento urbano

Fuente: Anisótropas Gomez, 2013 - Inventario Minero Lozano, 2008 actualizado ANM, 2013 (títulos mineros) Cartografía Base UAECD, 2013 
de estas etapas se diferencia por las características del tipo de vivienda y urbanísticas del asentamiento. (Castellanos, 2003, p.6)

"El patrón de asentamiento se encuentra asociado al origen del poblamiento que lo ha provocado. En este aspecto vale la pena señalar que una parte significativa de los barrios de origen ilegal de la localidad, iniciaron el poblamiento y por ende el establecimiento de los asentamientos, a partir de la existencia de la minería. En estos casos, se considera que el asentamiento se origina como "núcleo" desde una oferta de trabajo de carácter extractivo. Posteriormente, los barrios, a medida que crecen, paulatinamente tienden a tomar una configuración espacial definida que puede ser "sinuosa" en zonas de pendientes fuertes". (Camargo, 2005).

También se presenta el patrón de asentamiento "lineal" con referencia a la vía principal, pero con una configuración reticular en su interior, que se establece a partir de la avenida Boyacá y la avenida Villavicencio, como ejes principales, y en el interior de cada barrio se identifican las vías que son el eje del asentamiento. La mayoría de los asentamientos se han establecido en áreas pendientes, como producto del tipo de loteo que se realiza sobre los predios de mediana y gran extensión. (Adaptado de Castellanos, 2003)
La ontogenia en los barrios de origen ilegal en el área de la Localidad de Ciudad Bolívar es ocasionada:

- Por la fragmentación de un predio de mayor extensión ubicado inicialmente en el área rural cercana a la zona urbana.

- Alrededor de zonas dedicadas a la minería (canteras y chircales) y posterior reemplazamiento de estas ya sea porque son abandonadas o por la presión urbana.

- En las zonas aledañas a la infraestructura vial principal.

Según los materiales predominantes en las viviendas de la localidad, como las paredes exteriores, del total de hogares (145.353), el $22.1 \%$ (32.116) tiene ladrillo a la vista; el $14,3 \%$, bloque a la vista; el 58,8 \% , ladrillo o bloque revocado, pañetado o repellado. En viviendas de madera burda, tablón o tabla, habita el 1,5 $\%$ de los hogares y el 3,2 \% restante, en viviendas de otros materiales como piedra, madera pulida, tapia pisada, adobe, bahareque, material prefabricado, guadua, caña, esterilla, otro vegetal, zinc, tela, lona, cartón, latas, desechos, plástico y viviendas sin paredes (DANE, 2005).

\section{Resultados y conclusiones}

- La localización de la actividad minera en la zona de estudio se dio gracias al potencial minero para la 
extracción y transformación de materiales de construcción (agravas, arenas y arcillas).

- Las áreas de explotaciones mineras en la localidad, a lo largo del periodo de análisis (1970-2010), presentan relación con la urbanización ilegal considerando el origen de algunos de los barrios que actualmente conforman la localidad.

- El proceso de desplazamiento de las actividades mineras (generalmente minas abandonas o de baja producción) por los usos urbanos es muy dinámico y la urbanización de estas áreas obedece en la gran mayoría de los casos a factores como la tenencia de la tierra y la presión por la obtención de vivienda.

- El análisis de la evolución de las áreas construidas en los cuatro momentos (décadas 1970 a 2010), determinó que a medida que la actividad minera disminuye o desaparece es reemplazada por barrios de origen informal, los que después son incorporados al perímetro urbano mediante diversas estrategias o políticas.

- Entre 1970 y 1990 se muestra una tendencia al crecimiento de los desarrollos de origen ilegal, en torno a la actividad minera y las vías asociadas a esta, al igual que hacia el borde urbano de la ciudad, lo que indica un incremento en densidad en los bordes urbanos y la ocupación progresiva de lotes disponibles entre asentamientos.

- En las décadas siguientes la tendencia cambia, pues son los barrios los que configuran la distribución de la minería confinándola a las partes altas e incluso fuera de la ciudad.

- La organización espacial generada por la implantación, desarrollo y consolidación de los barrios de origen ilegal sobre antiguas canteras o áreas próximas a la actividad minera, dificulta la integración con las redes viales; por consiguiente, también es débil la conectividad y la accesibilidad con su entorno (localidades vecinas).

- El área urbana desarrollada en las zonas de montaña tiene restricciones en las conexiones, tanto con la zona urbana plana como con el resto de la ciudad.

- La zona de estudio presenta debilidades en el manejo y prevención de emergencias asociadas a la actividad minera, en el interior de las explotaciones y en las zonas circunvecinas.

\section{Referencias}

Almandoz, A. (2003). Historiografía urbana en Latinoamérica: del positivismo al Postmodernismo. Diálogos, 7, 117-156. Recuperado de http://www.dhi.uem.br/ publicacoesdhi/ dialogos/volume01/vol7_atg2 .htm. 
Bachaqueros, F. E. (1998). Plan de manejo de ecosistemas estratégicos para las áreas rurales del Distrito Capital. Informe Técnico. Bogotá: Departamento Administrativo del Medio Ambiente - DAMA (actual SDA).

Camargo, G. (2005). Ciudad ecosistema introducción a la ecología urbana. Bogotá: Secretaría Distrital de Ambiente, Universidad Piloto de Colombia.

Camargo, G. \& Guerrero, G. A. (2005). Lineamientos técnicos para la declaratoria y gestión en zonas amortiguadoras. Colección Lineamientos para la Gestión en Parques Nacionales Naturales. Bogotá: Panamericana Formas e Impresos S.A

Capel, H., (1975, febrero-mayo). La definición de lo urbano. Estudios Geográficos, (138139), 265-301.

Castellanos, M., (2003) Análisis de los sistemas de alteridad del borde oriental de Bogotá. Plan de Ordenamiento y Manejo de los Cerros Orientales de Bogotá. Bogotá: Departamento Administrativo del Medio Ambiente - DAMA (actual SDA)

Departamento Administrativo Nacional de Estadística -DANE- (2005). Censo nacional. Recuperado de http://www.dane.gov.co/index.php/estadisticas-sociales/deficit-de-vivienda

Díaz, C. (2011). Metabolismo de la ciudad de Bogotá D. C.: una herramienta para el análisis de la sostenibilidad ambiental urbana. Bogotá: Universidad Nacional de Colombia, Facultad de Ciencias Económicas.

Fierro, J. (2007). Caracterización geológica y geomorfológica de los PMI en las localidades de Ciudad Bolivar, Usme y Tunjuelo como apoyo a las acciones de conformación, participación, socialización y concertación de los Parques Minero Industriales (PMI). Bogotá: Secretaría Distrital de Ambiente.

Franquet, J. (2007). Un modelo racional de organización territorial. Aplicación a Cataluña. Tesis doctoral. Escuela Técnica Superior de Arquitectura, Universidad Internacional de Cataluña, Barcelona, España.

Ingeominas. (2005). Mapa geológico de la Sabana de Bogotá, en escala 1:100.000. Memoria Técnica. Bogotá: Ingeominas.

Instituto Geográfico Agustín Codazzi -IGAC-. (2003). Estudio general de suelos y zonificación de tierras del departamento de Cundinamarca. (Tomo II). Bogotá: IGAC.

Lozano, D. (2008). Aproximación a la cuantificación de pasivos ambientales relacionados con la minería en la localidad de Ciudad Bolívar Bogotá D.C mediante el análisis multitemporal de fotografía aéreas e imágenes de satélite. Tesis de grado, Facultad de Ciencias Departamento de Geociencias, Universidad Nacional de Colombia, Bogotá.

Luhmann, N. (1998). Sistemas sociales lineamientos para una teoría general. Bogotá: Anthropos, Universidad Iberoamericana, CEJA Pontificia Universidad Javeriana.

Massiris, A. (2005). Fundamentos conceptuales y metodológicos del ordenamiento territorial. En Colombia 2005. (p. 121). Bogotá: Jotamar.

Montoya, J. (2005). Teorías de la urbanización. Bogotá: Universidad Nacional de Colombia. 
Montoya, J. (2009). Geografía urbana: trayectorias epistemológicas y agenda contemporánea. En J. Montoya (ed.). Lecturas en teoría de la geografía. (pp. 149-187). Bogotá: Universidad Nacional de Colombia, Facultad de Ciencias Humanas.

Morín, E. (1999). El método: la naturaleza de la naturaleza. Madrid: Cátedra.

Ortiz, P. (2004). Conceptos teorías y métodos de la investigación geográfica. Teorías del análisis espacial y organización del espacio. Lecturas complementarias de varios autores. Traducción de la autora con base en documento Les Mots de la Géographie. Groupe GIP Reclus, Maison de La Géographie, Montpellier - Francia, 1997.

Rayo, L., (2008). Geología y geomorfología de la zona del parque minero industrial Tunjuelo. Bogotá: Secretaria Distrital de Ambiente.

Secretaría Distrital de Ambiente -SDA- (2007). Atlas ambiental de Bogotá D.C. Bogotá: Imprenta Nacional de Colombia. 272 p.

Secretaría Distrital de Ambiente -SDA- \& Universidad Nacional de Colombia (2008). Convenio 040 de 26 de Junio de 2007. Aunar esfuerzos técnicos, humanos, administrativos y económicos para la formulación del Plan de Ordenamiento y Manejo de la Cuenca del Río Tunjuelo en el perímetro urbano de Bogotá. (9 tomos). Informe Técnico. Bogotá: SDA.

Secretaría Distrital de Planeación -SDP- (2007). "Síntesis de la Problemática de las Áreas Desarrolladas Informalmente”. Dirección de Legalización y Mejoramiento Integral de Barrios (Contrato No. 0172 de 2007). Informe Técnico. Bogotá: SDP

Secretaría Distrital de Planeación -SDP- (2011). Monografías de las localidades: diagnóstico de los aspectos físicos, demográficos y socioeconómicos de las localidades - No. 19 Ciudad Bolívar. Bogotá: SDP. 
Recepción: 13 de noviembre de 2013

Evaluación: 20 de noviembre de 2013

Aprobación: 10 de diciembre de 2013 